\title{
Use of inflatable penile prostheses AMS CX with momentary squeeze in a patient with Peyronie's disease after removal of two previously implanted penile prostheses
}

\author{
Patrizio Vicini ${ }^{1}$, Ferdinando De Marco ${ }^{1}$, Gabriele Antonini ${ }^{2}$, Ettore De Berardinis ${ }^{2}$, \\ Riccardo Giovannone ${ }^{2}$, Stefano Pecoraro ${ }^{3}$, Luigi Azzarri ${ }^{1}$, Vincenzo Gentile ${ }^{2}$ \\ ${ }^{1}$ Department of Urology, "I.N.I." Italian Neurotraumatologic Institute Grottaferrata, Rome, Italy; \\ 2 Department of Urology, "Sapienza" Rome University, Rome, Italy; \\ ${ }^{3}$ Department of Urology, Malzoni Institute Avellino, Italy.
}

\begin{abstract}
Summary Objective: Peyronie's disease (PD) is a fibrotic wound-healing condition of the tunica albuginea that results in penile deformity, curvature, hinging, narrowing and shortening, penile pain, and in some cases, erectile dysfunction (ED). Surgery remains the gold standard treatment option, ensuring the faster and trustworthy treatment. For those patients who have erectile dysfunction and $P D$, penile prosthesis placement with straightening procedure is the best method to solve both diseases. The aim of this article is to present the use of hydraulic penile prostheses AMS CX with Momentary Squeeze associated with a complete isolation of the neurovascular bundle in a complex case after removal of two previously implanted prostheses in a man suffering from Peyronie's disease and erectile dysfunction.

Material and method: A 50 year-old patient underwent two previous prosthetic implants in another hospital. The first implantation was performed using an infrapubic approach followed by placement of a three-component hydraulic penile prosthesis. After six months the prosthesis was removed using an infra-pubic approach and two soft prosthesis Virilis II were implanted during the same surgery. One year after the second operation we implanted a hydraulic penile prosthesis AMS CX with Momentary Squeeze after complete isolation of the neurovascular bundle, fixing the two crural tips at the same level of albuginea of the two corpora cavernosa.

Result: Twelve months after surgery the penis was completely straight without penile shortening and the patient was fully satisfied with his sexual life.

Conclusion: The procedure enabled a perfect alignment of the cylinders along the longitudinal axis and penile prosthetic symmetry to obtain a good penile rigidity and a perfect penile straightening.
\end{abstract}

KEY WORDS: Inflatable penile prosthesis; Peyronie's disease; Erectile dysfunction; Isolation of the neurovascular bundle.

Submitted 17 September 2013; Accepted 5 October 2013

No conflict of interest declared

\section{INTRODUCTION}

Peyronie's disease (PD) is a fibrotic wound-healing condition of the tunica albuginea that results in penile deformity, curvature, hinging, narrowing and shortening, penile pain, and in some cases, erectile dysfunction (ED) (1-3). Although a lot of non-surgical options have been proposed, none to date offers a trustworthy and effective correction of the penile curvature. As a result, surgery remains the gold standard treatment option, ensuring the faster and trustworthy treatment (4).

Tunica albuginea plication is the recommended method of straightening for patients with adequate rigidity and less severe deformity described as curvature less than $70^{\circ}$ without narrowing/hinging (5-7). Patients who have more serious, complex Peyronie's disease, but maintain good preoperative erectile function should be submitted to a straightening consisting in plaque incision or partial excision and grafting (5-7). In the end, for those patients who have erectile dysfunction and $\mathrm{PD}$, penile prosthesis placement with straightening procedure is the best method to solve both diseases $(5,6,8,9)$.

The aim of this article is to present the use of hydraulic penile prostheses AMS CX with Momentary Squeeze associated with a complete isolation of the neurovascular bundle in a complex case after removal of two previous prostheses in a man suffering from PD and ED. After insertion of two crural tips, both tips have been attached symmetrically at the same level of the albuginea of the two corpora cavernosa. This has enabled a perfect alignment of the cylinders along the longitudinal axis and penile prosthetic symmetry to obtain a good penile rigidity and a perfect penile straightening.

Case Report and Figures are posted in Suppementary materials on www.aiua.it.

\section{Discussion}

The first and the second surgery did not correct penile curvature as the isolation of neurovascular bundle was 
not done. Before surgery we planned to make a geometrical incision of relaxation as well as application of a-cellular collagen matrix graft in order to allow better lengthening and straightening of the penis; this was not necessary, as the complete isolation of neurovascular bundle has allowed a good penile straightening by itself. We performed an apical dilation of the right corpus cavernosum to correctly reposition the right prosthetic cylinder, we fixed the crural tips of the two prosthetic cylinders with Prolene $2 / 0$ at the same crural level in order to obtain the alignment of the cylinders along the longitudinal axis and the symmetry of both cylinders of penile prosthesis (Figures 4-5).

As described in literature, the most common postoperative complaint from men who have undergone the penile implant is the length loss, for these reason we suggest an early activation of penile prostheses to avoid penile shortening (10-11).

\section{References}

1. Rosen R, Catania J, Lue T, et al. Impact of Peyronie's disease on sexual and psychological functioning: qualitative findings in patients in patients and controls. J Sex Med. 2008; 5:1997-84.

2. Smith JF, Walsh TJ, Conti S, et al. Risk factors for emotional and relationship problems in Peyronie's disease. J Sex Med. 2008; 5: 2179-84.
3. El-Sakka AI, Hassoba HM, Chui RM, et al. An animal model of Peyronie's like condition associated with an increase of transforming growth factor beta mRNA and protein expression. J Urol. 1997; 158:2284-90.

4. Larsen SM, Levine LA. Review of non surgical treatment options for Peyronie's disease. Int J Impot Res. 2012; 24:1-10.

5. Mulhall J, Anderson M, Parker M. A surgical algorithm for men with combined Peyronie's disease and erectile dysfunction. Functional and satisfaction outcomes. J Sex Med. 2005; 2:132-8.

6. Levine LA, Lenting EL. A surgical algorithm for the treatment of Peyronie's disease. J Urol. 1997; 158:2149-52.

7.Ralph DJ, Minhas S. The management of Peyronie's disease BJU Int. 2004; 93:208-15.

8. Mulhall J, Anderson M, Parker M. A surgical algorithm for men with combined Peyronie's disease and erectile dysfunction. Functional and satisfaction outcomes. J Sex Med. 2005; 2:132-8

9. Levine LA, Dimitriou RJ, A surgical algorithm for penile prosthesis placement in men with erectile failure and Peyronie's disease. Int J impot Res. 2000; 12:147-51.

10. Montague DK. Penile prostheses implantation: size matters. Eur Urol. 2007; 51:887-8.

11. Wang R, Howard GE, Hoang A, et al. Prospective and long-term evaluation of erectile penile length obtained with inflatable penile prostheses to that induced by intracavernosal injection. Asian J Androl. 2009; 411-5.

\section{Correspondence}

Patrizio Vicini, MD (Corresponding Author)

patriziovicini@gmail.com

Ferdinando De Marco, MD

Luigi Azzarri, MD

Department of Urology, "I.N.I." Italian Neurotraumatologic Institute Grottaferrata, Rome, Italy

Gabriele Antonini, MD

Ettore De Berardinis, MD

Riccardo Giovannone, MD

Vincenzo Gentile, MD

Department of Urology, "Sapienza" Rome University, Rome, Italy

Stefano Pecoraro, MD

Department of Urology, Malzoni Institute Avellino, Italy 